\title{
Article
}

\section{Allergy Attenuation by Cocoa-Enriched Diets}

\section{Margarida Castell 1,2,*}

1 Secció de Fisiologia, Departament de Bioquímica i Fisiologia, Facultat de Farmàcia i Ciències de l'Alimentació, Universitat de Barcelona (UB), 08028 Barcelona, Spain

2 Institut de Recerca en Nutrició i Seguretat Alimentària (INSA-UB), UB, 08921 Santa Coloma de Gramenet, Spain

* Correspondence: margaridacastell@ub.edu

Published: 30 October 2020

\begin{abstract}
Cocoa, derived from the Theobroma cacao tree, contains, among other compounds, methylxanthines such as theobromine, and polyphenols mainly flavonoids belonging to the flavanol family. It has been reported that cocoa consumption is beneficial for cardiovascular and brain health, in the protection against cancer, but it remained to know what happen in allergic diseases. In this talk, I will explain the influence of a cocoa-enriched diet in two models of allergy in rats, including the effects on the anaphylactic response. Moreover, I will introduce the results using particular cocoa populations. Apart of the preclinical studies developed in rats, the results of an observational study in university students are included. In summary, although more studies are needed, it will be demonstrated the potential of cocoa intake in the prevention of allergic reactions.
\end{abstract}

Publisher's Note: MDPI stays neutral with regard to jurisdictional claims in published maps and institutional affiliations.

(C) 2020 by the authors. Submitted for possible open access publication under the terms and conditions of the Creative Commons Attribution (CC BY) license (http://creativecommons.org/licenses/by/4.0/). 